\title{
Visualizing Investment Decision on Decision Balls
}

\author{
Li-Ching Ma \\ Department of Information Management, National United University, Taipei \\ E-mail: lcma@nuu.edu.tw \\ Received May 10, 2011; revised May 30, 2011; accepted June 8, 2011
}

\begin{abstract}
Decision makers' choices are often influenced by visual background information. This study uses open-ended equity funds in Taiwan to investigate three well-known optimal portfolio models, including the mean-variance, maximin, and minimization of mean absolute deviation. The optimal portfolios are then visualized on Decision Balls to assist investors in making investment decisions. By observing the Decision Balls, investors can see the optimal portfolios, compare the optimal weights provided by the different models, view the cluster of funds, and even find substitute funds if preferred funds are not available.
\end{abstract}

Keywords: Visualization, Decision Ball, Investment decision, Portfolio

\section{Introduction}

Decision makers' choices are often influenced by visual background information [1,2]. Visual representations can simplify complex information into meaningful patterns, assist people in comprehending their environment, and allow for simultaneous perceptions of parts as well as the interrelationships between parts [3]. Visual representations are also recognized as being useful to present financial issues. For instance, the efficient frontier [4] is a well known visual representation used to help investors understand relationships between risks and returns.

Several graphic methods have been developed to support the decision-making: for instance, Gower Plots to detect any inconsistencies in a decision maker's preferences and rank alternatives [5,6], and ELECTRE graphs to help decision makers understand investment problems [7]. All these methods, however, use a 2-dimensional plane to illustrate the multidimensional data. A 2-dimensional plane model cannot depict three points that do not obey the triangular inequality (i.e. the total length of any two edges must be larger than the length of the third edge) neither can it display four points that are not on the same plane [8].

The method employed here, is the Decision Ball, which has not been used previously for visualizing portfolio. The Decision Ball method $[8,9]$ is based on multidimensional scaling (MDS) $[10,11]$ which has been widely used in marketing and decision-making $[12,13]$. This study extends the Decision Ball method to visualize optimal portfolios on the surface of a sphere. The dis- tance between two securities is used to represent the correlation between them: the larger the correlation, the shorter the distance. Also, the fund with the higher return is located closer to the North Pole. Mutual funds in Taiwan are taken as an example to demonstrate how to assist investors visualize optimal portfolios on the Decision Ball.

Taiwan's mutual fund industry, which was founded in 1983, has been growing tremendously during the last decade $[14,15]$, with the number of mutual fund corporations increasing from 4 to 38 by 2008. In 1998, there were only 200 funds with a total net asset value of NT $\$ 745.97$ billion. However, by January 2008, there were 523 funds with a net asset value totaling NT\$2,040.91 billion. This shows that the total net asset values of funds have almost tripled during the last decade. In Taiwan, the mutual fund industry is dominated by individual investors who account for over $90 \%$ of the market volume. By January 2008, over 1.84 million investors, about $8 \%$ of Taiwan's population, had invested in mutual funds.

This study examines 174 open-ended equity mutual funds which were issued and invested in Taiwan's Market from January 2002 to December 2006. Three wellknown optimal portfolio models, including the mean- variance [4], the maximin [16], and the minimization of mean absolute deviation [17], are investigated. The optimal portfolios are visualized on the Decision Balls. By studying the Decision Balls, investors can then see the optimal portfolios, compare the optimal weights provided by different models, view the cluster of funds, and even find substitute funds if the preferred funds are not available. 
This paper is organized as follows: Section 2 briefly reviews three well-known models for optimal portfolios. Section 3 develops an extended Decision Ball model to allocate funds on the surface of a sphere. Section 4 uses Taiwan's Open-Ended Equity Funds as an example to examine three optimal portfolio models, and Section 5 demonstrates how to visualize optimal portfolios on the Decision Balls.

\section{Optimization Models for Portfolio Problem}

Three well-known approaches to formulate optimal portfolios are illustrated in this section, including a) a meanvariable model denoted as MinVar, b) a maximin model denoted as MaxiMin, and c) a minimization of mean absolute deviation model denoted as MinMAD.

The mean-variance model, first proposed by Harry Markowitz, is a quadratic programming model to minimize the variance given a required return. Suppose there are $\mathrm{n}$ securities, the mean-variance model is formulated as follows:

\subsection{Mean-Variance Model (MinVar)}

$$
\begin{array}{ll}
\text { Min } & \operatorname{Var}=\sum_{i=1}^{n} \sum_{j=1}^{n} \sigma_{i, j} w_{i} w_{j} \\
\text { subject to: } & \sum_{i=1}^{n} w_{i}=1 \\
& \sum_{i=1}^{n} w_{i} u_{i} \geq a \\
& 0 \leq w_{i} \leq 1, \text { for all } \quad i
\end{array}
$$

where $w_{i}$ denotes the portfolio allocation of security $i$; $\sigma_{i, j}$ denotes the covariance between security $i$ and security $j ; \mu_{i}$ is the mean return for security $i ; \alpha$ is the minimum expected return required by a particular investor.

Two important assumptions of the mean-variance model are: the investor prefers a low risk; and the expected return is multivariate normally distributed. The mean-variance model has been widely used in various portfolio problems. However, it may take some time to find optimal solutions with a large number of securities because the objective function is quadratic.

The maximin model [16] is a linear programming model to maximize the minimum portfolio return required by an investor. Denoting $P$ as the minimum required return by an investor for every time period, $T$ as the total number of periods, and $r_{i, t}$ as the return for security $i$ over period $t$, where $t=1, \cdots, T$, the maximin linear model is formulated as follows:

\subsection{Maximin Linear Model (MaxiMin)}

$\begin{array}{ll}\text { Max } & P \\ \text { subject to: } & \sum_{i=1}^{n} r_{i, t} w_{i} \geq P, \forall t \\ & P \geq 0 \\ & (1),(2),(3)\end{array}$

Contrary to the mean-variance model to lower risk by minimizing the variance, the object of this model is to maximize the minimum return over a set of past returns. The major advantage of this model is its capability to deal with portfolio optimization problems involving a large number of securities. Also, according to Young [16], the maximin model is more appropriate than the mean-variance model when data is log-normally distributed or skewed. However, this model may lead to an infeasible solution if the sum of the weighted expected returns is negative for any period of time.

The minimization of mean absolute deviation model [17] is another alternative to simplify the mean-variance model. This model uses the mean absolute deviation as a risk measure. The mean absolute deviation is defined as: $\frac{1}{T} \sum_{t=1}^{T}\left|\sum_{i=1}^{n}\left(r_{i, t}-\mu_{i}\right) w_{i}\right|$, Let $Q_{t}=\left|\sum_{i=1}^{n}\left(r_{i, t}-\mu_{i}\right) w_{i}\right|$, the minimization of mean absolute deviation model can be linearized as a linear programming formulation as follows:

\subsection{Minimization of Mean Absolute Deviation Model (MinMAD)}

$$
\begin{array}{ll}
\operatorname{Min} & \frac{1}{T} \sum_{t=1}^{T} Q_{t} \\
\text { subject to: } & Q_{t} \geq-\sum_{i=1}^{n}\left(r_{i, t}-\mu_{i}\right) w_{i}, \quad \forall t \\
& Q_{t} \geq-\sum_{i=1}^{n}\left(r_{i, t}-\mu_{i}\right) w_{i}, \quad \forall t \\
& Q_{t} \geq 0, \forall t \\
& (1),(2),(3)
\end{array}
$$

The complexity of this model is much lower than that of a mean-variance model since the objective function is linear rather than quadratic. This model provides similar results as the mean-variance model if the return is multivariate normally distributed [17].

These three models have been examined by 67 securities over 48 months traded on the Stockholm Stock Exchange [18]. The results show that the maximin model provides the highest return and risk, the mean-variance model yields the lowest risk and return, and the result of the minimization of mean absolute deviation model is close to that of the mean-variance model. This study tries to use mutual funds in Taiwan to examine these three 
models and then visualize the results on Decision Balls.

\section{An Extended Decision Ball Model}

In order to visualize the relationships among funds, a Decision Ball model [8] is applied and extended here to display funds on the surface of a hemisphere.

The Decision Ball model is based on the concept of a multidimensional scaling technique. The arc length between two alternatives is used to represent the dissimilarity between them, e.g. the larger the difference, the longer the arc length. However, because the arc length is monotonically related to the Euclidean distance between two points and both approximation methods make little difference to the resulting configuration [19], the Euclidean distance is used for simplification purposes. Also, the alternative with a higher score value is designed to be closer to the North Pole so that alternatives will be located on the concentric circles in scoring order from top view.

In this study, the correlation coefficient is adopted to describe the degree of relationship between two funds because it is one of the most common statistics and it detects linear dependencies between two variables. The linear feature makes it easier to be visualized than covariance. Consider $n$ funds denoted as $A_{i}, i \in\{1, \cdots, n\}$. Denote $\rho_{i, j}$ as the correlation coefficient between securities $i$ and $j$, where $-1 \leq \rho_{i, j} \leq 1$ and $\rho_{i, j}=1$ for all $i, j$. The closer the coefficient is to either -1 or 1 , the stronger the correlation between the variables. If the variables are independent then the correlation is 0 .

The distance between two funds is used to represent the correlation between them, i.e. the larger the correlation, the shorter the distance. The Euclidean distance between $A_{i}$ and $A_{j}$ is denoted as $d_{i, j}$, and $\hat{d}_{i, j}$ as the mapped distance of correlation. The relationship between $\hat{d}_{i, j}$ and $\rho_{i, j}$ is defined as below:

$$
\hat{d}_{i, j}=s \times\left(1-\rho_{i, j}\right),
$$

where $s$ is a scaling constant. It is obvious $\hat{d}_{i, j}=\hat{d}_{j, i}$.

The scaling constant can be given as

$$
s=\left\{\begin{array}{c}
\sqrt{2} / \operatorname{Max}\left\{1-\rho_{i, j}\right\}, \text { if } \operatorname{Max}\left\{1-\rho_{i, j}\right\} \neq 0, \forall i, j . \\
\sqrt{2}, \text { if } \operatorname{Max}\left\{1-\rho_{i, j}\right\}=0, \forall i, j .
\end{array}\right.
$$

In Expression (10), $\sqrt{2}$ is used because the distance between the North Pole and the Equator is $\sqrt{2}$ when the radius $=1$. From Expressions (9) and (10), if $\rho_{i, j}=$ 1 then $\hat{d}_{i, j}=0$; if $\rho_{i, j}=0$ then $\hat{d}_{i, j}=s$; if $\rho_{i, j}=-1$ then $\hat{d}_{i, j}=2 \times s=\sqrt{2}$. That is, the larger the correlation, the shorter the distance. The range of $\hat{d}_{i, j}$ is $0 \leq \hat{d}_{i, j}$ $\leq \sqrt{2}$.

The fund with the higher return is designed to be located closer to the North Pole. The coordinates of a fund Ai are denoted on a ball as $\left(x_{i}, y_{i}, z_{i}\right)$. Given a radius $=1$, the coordinate of the North Pole is expressed as $(0,1,0)$. An extended Decision Ball model for portfolio selection is formulated as follows:

Model 1 (An Extended Decision Ball Model for Portfolio Selection)

$$
\operatorname{Min}_{\left\{x_{i}, y_{i}, z_{i}\right\}} \sum_{i=1}^{n} \sum_{j>i}^{n}\left(d_{i, j}-\hat{d}_{i, j}\right)^{2}
$$

Subject to

$$
\begin{aligned}
& \hat{d}_{i, j}=s \times\left(1-\rho_{i, j}\right), \quad \forall i, j>i, \\
& y_{i} \geq y_{j}, \text { if } \mu_{i} \geq \mu_{j}, \quad \forall i, j, \\
d_{i, j}^{2}= & \left(x_{i}-x_{j}\right)^{2}+\left(y_{i}-y_{j}\right)^{2}+\left(z_{i}-z_{j}\right)^{2}, \quad \forall i, j>i, \\
& x_{i}^{2}+y_{i}^{2}+z_{i}^{2}=1, \quad \forall i, \\
- & 1 \leq x_{i} \leq 1, \quad 0 \leq y_{i} \leq 1, \quad-1 \leq z_{i} \leq 1, \quad \forall i
\end{aligned}
$$

The objective of Model 1 is to minimize the sum of the squared differences between $d_{i, j}$ and $\hat{d}_{i, j}$. Constraint (11) is from Expression (9). Constraint (12) is designed for the fund with a higher return to be located closer to the North Pole. Euclidean distance, instead of arc length, is used for simplification purposes (13). All alternatives are graphed on the surface of a sphere (14) and located on the northern hemisphere (15).

The faithfulness of this visual representation can be measured by Stress [20], which is a numerical measure of the closeness between the dissimilarities in the lower dimension and the original spaces formulated as follows:

$$
\text { Stress }=\sqrt{\frac{\sum_{i=1}^{n} \sum_{j>i}^{n}\left(d_{i, j}-\hat{d}_{i, j}\right)^{2}}{\sum_{i=1}^{n} \sum_{j>i}^{n} d_{i, j}^{2}}}
$$

A solution is desirable if its stress value is less than $10 \%$.

Model 1 is a nonlinear model, which can be solved by using some commercial optimization software, such as Global Solver of Lingo 9.0, to obtain an optimum solution. This model has good performance results when the number of funds is small. However, when $\mathrm{n}$ becomes large, the computational time will increase greatly since the time complexity of Model 1 is $n^{2}$. In practice, in the case of more than 10 funds, we can choose some target funds as anchor points. The coordinates of the anchor 
points are calculated first, and then the coordinates of the remaining funds can be obtained by calculating the correlations between those funds and the anchor points. Thus, all funds can be displayed on the Decision Ball within a tolerable time frame.

\section{Empirical Study of Taiwan Open-Ended Equity Funds}

This study takes 174 open-ended equity funds, which were issued and invested in the Taiwan Market from January 2002 to December 2006 for example to investigate the three optimization models. From these 174 funds, 39 funds are excluded for the following two reasons: 1) 9 funds were not listed at the starting period 2) 30 funds left for various reasons over the examined time period. The monthly returns and rankings of the 135 open-ended equity funds are listed in Table 1. Fund number 129 has the highest monthly return of 0.0170 ; whereas, fund number 46 yields the lowest return, -0.0014 . The average monthly return of the 135 funds is 0.0078 .

To simplify, suppose our investors are only interested in the top 30 performance funds (i.e., rank $1 \sim 30$ ) and request monthly returns of at least $1 \%$, and short selling is not allowed. The descriptive statistics for the top 30 funds are listed in Table 2. The third, fourth, fifth, and sixth columns of Table 2 show the mean of the monthly return, the standard deviation, and both the minimum and maximum values of the funds. The last column describes the ranking of funds.

The MinVar, MaxiMin, and MinMAD models are all examined using the top 30 funds. However, the MaxiMin model yields infeasible solutions. The reason is that all top 30 funds exhibited negative monthly returns in some months. For instance, in September 2002, affected by that year's stock market downturn across the United States, Europe, and Asia, the average monthly return of the top 30 funds was -0.0892 ranging from -0.1242 to -0.0577 .

The results of the MinVar and MinMAD models are listed in Table 3. Only those funds which appear in portfolios at least once, i.e. the funds numbering 12, 86, 129, and 133, are shown. The weights of the funds in an optimal portfolio for four different $\alpha$ are exhibited. Because $\alpha$ ranging from $1 \%$ to $1.3 \%$ yields the same portfolio weights, $1.0 \leq \alpha \leq 1.3 \%$ is presented for short. Also, since the maximum mean of monthly returns for the top 30 funds is 0.0170 (exactly, 0.01695 ), $\alpha \geq 1.7 \%$ is neglected because none of top 30 funds yields the mean of monthly returns greater than or equal to $1.7 \%$. The bottom two rows of Table 3 indicate the portfolio return and variance.

As shown in Table 3, the portfolio weights in both the MinVar and MinMAD models remain unchanged for low $\alpha$ values ranging from $1.0 \%$ to $1.3 \%$. In this range, the
MinVar model yields an optimal portfolio return of 0.0132 , a variance of 0.0023 , and weights $w_{86}=0.053$, $w_{129}=0.243$, and $w_{133}=0.704$. Whereas, the MinMAD model yields an optimal portfolio return of 0.0130 , a portfolio variance of 0.0023 , and portfolio weights $w_{129}=$ 0.219 and $w_{133}=0.781$. As we can see, the MinVar model provides a higher expected return than required, and higher also than that of the MinMAD model. Given $\alpha=1.4 \%$, the MinVar and MinMAD models yield exactly the same solutions with a portfolio return of 0.014 , a variance of 0.0024 , and with weights $w_{129}=0.413$ and $w_{133}=0.587$. Given $\alpha=1.5 \%$ and $1.6 \%$, an optimal portfolio of the MinVar and MinMAD models consists of the same funds with different weights. The expected portfolio return and variance are the same.

In this study, the outcome of the MinMAD model is quite close to that of the MinVar model. This result is the same as the conclusions of Papahristodoulou and Dotzauer [18], in which 67 shares traded on the Stockholm Stock Exchange between January 1997 and December 2000 were examined. Both models provide optimal portfolio suggestions. However, the investors cannot tell directly, the correlations among funds through table- listing. The next section will demonstrate how to visualize the optimal portfolio on Decision Balls.

\section{Visualizing Optimal Portfolios on Decision Balls}

An extended Decision Ball model uses the distance between two funds to represent the correlation between them, i.e. the larger the correlation, the shorter the distance. Also, the fund with the higher return is located closer to the North Pole. At first, a correlation matrix of funds is calculated. From Expression (9), $\hat{d}_{i, j}$ for all $i, j$ can be calculated. Since $\operatorname{Max}\left\{1-\rho_{i, j}\right\}=0.3064$ for all $i, j$, from Expression (10), the scaling constant $\mathrm{s}$ is given as 4 in this example. If the number of funds being considered is small, then Model 1 can be applied directly to yield the coordinates of all funds. However, when the number of funds is large, the computational time for Model 1 will increase greatly.

In order to increase computational efficiency, the four funds listed in Table 3, i.e. the funds numbered 12, 86, 129 , and 133, can be chosen as the target funds. These four funds, in which investors may be the most interested, are suggested in an optimal portfolio for both the MinVar and MinMAD models. The correlation matrix of the target funds is calculated first. Applying Model 1 to these four funds yields the coordinates of them, the so called anchor points. The coordinates of funds numbered 12, 86, 129, and 133 are $\{0.820,0.565,0.083\},\{0.292,0.365,0.883\}$, 
Table 1. The expected monthly returns from 135 mutual funds.

\begin{tabular}{|c|c|c|c|c|c|c|c|c|c|c|c|}
\hline No & Quote & Return & Rank & No & Quote & Return & Rank & No & Quote & Return & Rank \\
\hline 1 & 0001 & 0.00095 & 133 & 46 & IC25 & -0.00143 & 135 & 91 & ML09 & 0.01017 & 38 \\
\hline 2 & 0002 & 0.00807 & 63 & 47 & II01 & 0.00557 & 93 & 92 & $\mathrm{NC} 13$ & 0.00613 & 87 \\
\hline 3 & 0003 & 0.00523 & 100 & 48 & II05 & 0.00882 & 53 & 93 & YC07 & 0.00276 & 124 \\
\hline 4 & 0004 & 0.00775 & 67 & 49 & II13 & 0.00715 & 74 & 94 & ML15 & 0.01092 & 30 \\
\hline 5 & 0005 & 0.00883 & 52 & 50 & JF51 & 0.00385 & 114 & 95 & UI05 & 0.00261 & 126 \\
\hline 6 & 0012 & 0.00258 & 127 & 51 & JS01 & 0.00533 & 96 & 96 & AI05 & 0.00528 & 99 \\
\hline 7 & 0013 & 0.00327 & 120 & 52 & JS03 & 0.01424 & 7 & 97 & BR05 & 0.00870 & 55 \\
\hline 8 & 0014 & 0.01243 & 18 & 53 & KG01 & 0.00893 & 50 & 98 & CA09 & 0.01222 & 22 \\
\hline 9 & 0017 & 0.01171 & 27 & 54 & KY01 & 0.01580 & 2 & 99 & CI07 & 0.00422 & 109 \\
\hline 10 & 0018 & 0.00353 & 117 & 55 & ML01 & 0.01414 & 8 & 100 & СР03 & 0.00962 & 41 \\
\hline 11 & 0021 & 0.01013 & 39 & 56 & ML02 & 0.00571 & 91 & 101 & CP14 & 0.01071 & 33 \\
\hline 12 & 0025 & 0.01551 & 3 & 57 & ML06 & 0.01053 & 35 & 102 & CS07 & 0.00465 & 107 \\
\hline 13 & 0026 & 0.00987 & 40 & 58 & ML12 & 0.00688 & 77 & 103 & DD04 & 0.00639 & 82 \\
\hline 14 & AI01 & 0.00508 & 103 & 59 & $\mathrm{NC} 03$ & 0.00684 & 78 & 104 & DF07 & 0.00752 & 69 \\
\hline 15 & AP01 & 0.00367 & 115 & 60 & $\mathrm{NC} 07$ & 0.00626 & 84 & 105 & DS03 & 0.00239 & 128 \\
\hline 16 & AP04 & 0.00684 & 79 & 61 & NC09 & 0.00858 & 57 & 106 & FH06 & 0.01215 & 24 \\
\hline 17 & BR01 & 0.00730 & 73 & 62 & PS01 & 0.00652 & 81 & 107 & FP11 & 0.00587 & 90 \\
\hline 18 & CA03 & 0.00917 & 46 & 63 & PS02 & 0.00706 & 76 & 108 & FP16 & 0.00804 & 64 \\
\hline 19 & CA04 & 0.00396 & 112 & 64 & PS03 & 0.00959 & 43 & 109 & GC05 & 0.01235 & 20 \\
\hline 20 & CF01 & 0.00887 & 51 & 65 & PS09 & 0.00626 & 85 & 110 & GC17 & 0.00626 & 86 \\
\hline 21 & CI01 & 0.00514 & 102 & 66 & PS14 & 0.01408 & 9 & 111 & IC06 & 0.00708 & 75 \\
\hline 22 & CI04 & 0.00523 & 101 & 67 & TC01 & 0.00896 & 49 & 112 & IC30 & 0.00283 & 123 \\
\hline 23 & CI05 & 0.00823 & 61 & 68 & TI02 & 0.00761 & 68 & 113 & II11 & 0.00961 & 42 \\
\hline 24 & CI12 & 0.00410 & 111 & 69 & TI07 & 0.01281 & 16 & 114 & JF76 & 0.00357 & 116 \\
\hline 25 & CP04 & 0.00423 & 108 & 70 & TR01 & 0.00208 & 130 & 115 & JS07 & 0.00589 & 89 \\
\hline 26 & CP07 & 0.00264 & 125 & 71 & TS02 & 0.00286 & 122 & 116 & KY06 & 0.00920 & 45 \\
\hline 27 & $\mathrm{CS} 02$ & 0.00783 & 65 & 72 & TS08 & 0.00839 & 59 & 117 & ML07 & 0.00294 & 121 \\
\hline 28 & CS09 & 0.00531 & 97 & 73 & TS13 & 0.00680 & 80 & 118 & $\mathrm{NC} 16$ & 0.01322 & 13 \\
\hline 29 & CT01 & 0.00735 & 72 & 74 & YT02 & 0.01329 & 12 & 119 & PS10 & 0.01076 & 31 \\
\hline 30 & CY01 & 0.00873 & 54 & 75 & YT03 & 0.00904 & 48 & 120 & TC03 & 0.00567 & 92 \\
\hline 31 & CY14 & 0.00239 & 129 & 76 & YT04 & 0.01244 & 17 & 121 & TC19 & 0.01317 & 14 \\
\hline 32 & DD01 & 0.00530 & 98 & 77 & YT11 & 0.01538 & 4 & 122 & TR04 & 0.00590 & 88 \\
\hline 33 & DS04 & 0.00115 & 132 & 78 & YT12 & 0.01370 & 10 & 123 & TS09 & 0.00741 & 71 \\
\hline 34 & FD01 & 0.00534 & 95 & 79 & 0023 & 0.01158 & 28 & 124 & UI03 & -0.00048 & 134 \\
\hline 35 & FE01 & 0.00638 & 83 & 80 & 0029 & 0.00467 & 106 & 125 & $\mathrm{YC} 02$ & 0.00782 & 66 \\
\hline 36 & FH01 & 0.00351 & 118 & 81 & BR03 & 0.00853 & 58 & 126 & YT09 & 0.00911 & 47 \\
\hline 37 & FH03 & 0.00868 & 56 & 82 & CI11 & 0.00500 & 105 & 127 & 0015 & 0.00507 & 104 \\
\hline 38 & FP03 & 0.01223 & 21 & 83 & CP10 & 0.01143 & 29 & 128 & FP05 & 0.01213 & 25 \\
\hline 39 & FP04 & 0.01059 & 34 & 84 & CY03 & 0.01479 & 5 & 129 & JS06 & 0.01695 & 1 \\
\hline 40 & FP06 & 0.01076 & 32 & 85 & DF05 & 0.00416 & 110 & 130 & TI09 & 0.00386 & 113 \\
\hline 41 & FP10 & 0.01450 & 6 & 86 & FH08 & 0.01218 & 23 & 131 & FD02 & 0.00952 & 44 \\
\hline 42 & GC01 & 0.00829 & 60 & 87 & $\mathrm{IC} 08$ & 0.01043 & 36 & 132 & FP15 & 0.01334 & 11 \\
\hline 43 & $\mathrm{IC} 01$ & 0.00538 & 94 & 88 & II10 & 0.00329 & 119 & 133 & JF85 & 0.01192 & 26 \\
\hline 44 & $\mathrm{IC} 04$ & 0.00743 & 70 & 89 & JF83 & 0.00821 & 62 & 134 & $\mathrm{NC} 17$ & 0.01285 & 15 \\
\hline 45 & $\mathrm{IC} 22$ & 0.00185 & 131 & 90 & JS04 & 0.01243 & 19 & 135 & YT16 & 0.01031 & 37 \\
\hline
\end{tabular}

Table 2. Descriptive statistics for the top $\mathbf{3 0}$ mutual funds.

\begin{tabular}{|c|c|c|c|c|c|c|c|c|c|c|c|c|c|}
\hline No & Quote & Return & STD & Min & Max & Rank & No & Quote & Return & STD & Min & Max & Rank \\
\hline 8 & 0014 & 0.0124 & 0.0662 & -0.1157 & 0.1625 & 18 & 83 & CP10 & 0.0114 & 0.0626 & -0.1214 & 0.1508 & 29 \\
\hline 9 & 0017 & 0.0117 & 0.0614 & -0.1118 & 0.1472 & 27 & 84 & CY03 & 0.0148 & 0.0587 & -0.1157 & 0.1379 & 5 \\
\hline 12 & 0025 & 0.0155 & 0.0573 & -0.1118 & 0.1426 & 3 & 86 & FH08 & 0.0122 & 0.0591 & -0.1366 & 0.1951 & 23 \\
\hline 38 & FP03 & 0.0122 & 0.0632 & -0.1279 & 0.1529 & 21 & 90 & JS04 & 0.0124 & 0.0731 & -0.1405 & 0.241 & 19 \\
\hline 41 & FP10 & 0.0145 & 0.0672 & -0.123 & 0.1985 & 6 & 94 & ML15 & 0.0109 & 0.0721 & -0.1263 & 0.2299 & 30 \\
\hline 52 & JS03 & 0.0142 & 0.072 & -0.1224 & 0.1905 & 7 & 98 & CA09 & 0.0122 & 0.0695 & -0.1418 & 0.1755 & 22 \\
\hline 54 & KY01 & 0.0158 & 0.071 & -0.1139 & 0.1478 & 2 & 106 & FH06 & 0.0121 & 0.0573 & -0.096 & 0.1554 & 24 \\
\hline 55 & ML01 & 0.0141 & 0.0674 & -0.1097 & 0.1909 & 8 & 109 & GC05 & 0.0123 & 0.0664 & -0.1297 & 0.1663 & 20 \\
\hline 66 & PS14 & 0.0141 & 0.0679 & -0.1141 & 0.2176 & 9 & 118 & NC16 & 0.0132 & 0.0694 & -0.1325 & 0.1995 & 13 \\
\hline 69 & TI07 & 0.0128 & 0.0688 & -0.1091 & 0.1728 & 16 & 121 & TC19 & 0.0132 & 0.0673 & -0.1252 & 0.1621 & 14 \\
\hline 74 & YT02 & 0.0133 & 0.0654 & -0.1131 & 0.1948 & 12 & 128 & FP05 & 0.0121 & 0.0687 & -0.1219 & 0.2403 & 25 \\
\hline 76 & YT04 & 0.0124 & 0.0658 & -0.1486 & 0.1709 & 17 & 129 & JS06 & 0.017 & 0.0545 & -0.0898 & 0.1418 & 1 \\
\hline 77 & YT11 & 0.0154 & 0.0608 & -0.1042 & 0.1757 & 4 & 132 & FP15 & 0.0133 & 0.0712 & -0.1271 & 0.1989 & 11 \\
\hline 78 & YT12 & 0.0137 & 0.0723 & -0.1359 & 0.2337 & 10 & 133 & JF85 & 0.0119 & 0.0494 & -0.1108 & 0.1099 & 26 \\
\hline 79 & 0023 & 0.0116 & 0.0618 & -0.1202 & 0.1698 & 28 & 134 & $\mathrm{NC} 17$ & 0.0128 & 0.0616 & -0.1109 & 0.1739 & 15 \\
\hline
\end{tabular}


Table 3. Portfolios of the top 30 mutual funds by the MinVar and MinMAD models.

\begin{tabular}{cccccccccc}
\hline & & \multicolumn{2}{c}{$1.0 \% \leq a \leq 1.3 \%$} & \multicolumn{2}{c}{$a=1.4 \%$} & \multicolumn{2}{c}{$a=1.5 \%$} & \multicolumn{2}{c}{$a=1.6 \%$} \\
\hline No & Quote & MinVar & MinMAD & MinVar & MinMAD & MinVar & MinMAD & MinVar & MinMAD \\
12 & 0025 & 0 & 0 & 0 & 0 & 0.084 & 0.165 & 0.216 & 0.298 \\
86 & FH08 & 0.053 & 0 & 0 & 0 & 0 & 0 & 0 & 0 \\
129 & JS06 & 0.243 & 0.219 & 0.413 & 0.413 & 0.552 & 0.494 & 0.657 & 0.598 \\
133 & JF85 & 0.704 & 0.781 & 0.587 & 0.587 & 0.364 & 0.341 & 0.127 & 0.104 \\
P. Return & 0.0132 & 0.013 & 0.014 & 0.014 & 0.015 & 0.015 & 0.016 & 0.016 \\
\multicolumn{2}{r}{ P. Variance } & 0.0023 & 0.0023 & 0.0024 & 0.0024 & 0.0025 & 0.0025 & 0.0026 & 0.0026 \\
\hline
\end{tabular}

Table 4. Coordinates of the top 30 mutual funds.

\begin{tabular}{|c|c|c|c|c|c|c|c|c|c|}
\hline No & Quote & $x$ & $y$ & $Z$ & No & Quote & $x$ & $y$ & $Z$ \\
\hline 8 & 0014 & 0.854 & 0.415 & -0.312 & 83 & CP10 & 0.525 & 0.189 & 0.83 \\
\hline 9 & 0017 & 0.677 & 0.325 & 0.659 & 84 & CY03 & 0.476 & 0.545 & 0.689 \\
\hline 12 & 0025 & 0.82 & 0.565 & 0.083 & 86 & FH08 & 0.292 & 0.365 & 0.883 \\
\hline 38 & FP03 & 0.621 & 0.385 & 0.682 & 90 & JS04 & 0.085 & 0.405 & 0.911 \\
\hline 41 & FP10 & 0.503 & 0.535 & 0.678 & 94 & ML15 & 0.598 & 0.179 & 0.782 \\
\hline 52 & JS03 & 0.154 & 0.525 & 0.836 & 98 & CA09 & 0.56 & 0.375 & 0.738 \\
\hline 54 & KY01 & 0.59 & 0.575 & 0.566 & 106 & FH06 & 0.596 & 0.355 & 0.719 \\
\hline 55 & ML01 & 0.667 & 0.515 & 0.538 & 109 & GC05 & 0.545 & 0.395 & 0.739 \\
\hline 66 & PS14 & 0.597 & 0.505 & 0.622 & 118 & NC16 & 0.159 & 0.465 & 0.87 \\
\hline 69 & TI07 & -0.043 & 0.435 & 0.899 & 121 & TC19 & 0.449 & 0.455 & 0.768 \\
\hline 74 & YT02 & 0.486 & 0.475 & 0.733 & 128 & FP05 & 0.456 & 0.345 & 0.82 \\
\hline 76 & YT04 & 0.841 & 0.425 & -0.334 & 129 & JS06 & 0.183 & 0.856 & 0.483 \\
\hline 77 & YT11 & 0.616 & 0.555 & 0.558 & 132 & FP15 & 0.641 & 0.485 & 0.594 \\
\hline 78 & YT12 & 0.463 & 0.495 & 0.734 & 133 & JF85 & 0.914 & 0.335 & 0.227 \\
\hline 79 & 0023 & 0.507 & 0.199 & 0.838 & 134 & $\mathrm{NC} 17$ & 0.674 & 0.445 & 0.589 \\
\hline
\end{tabular}

$\{0.183,0.856,0.483\}$, and $\{0.914,0.335,0.227\}$ respectively. The coordinates for the remaining funds can be obtained by calculating the correlations between those funds and the target funds. The locations of all top 30 funds are listed in Table $\mathbf{4}$ and depicted in Figure 1.

In Figure 1, the four target funds are shown as bold font. Since the expected return is $\mu_{129}>\mu_{12}>\mu_{86}>\mu_{133}$, fund 129 is closest to the northern point followed by funds $12,86,133$. Also, the distance between the two funds is used to represent the correlation between them: the larger the correlation, the shorter the distance. Take fund 12 for example, because the correlation between funds 12 and $133\left(\rho_{12,133}=0.9087\right)$ is higher than that between funds 12 and $129\left(\rho_{12,129}=0.7501\right)$, then the distance between the former is shorter than that of the later.

The optimal portfolios obtained by the MinVar and MinMAD models are graphed on the Decision Ball and represented respectively with "dotted" and "dash" circles. The scale of the circle represents the weight of the fund: the higher the weight, the larger the circle. Given a required return $\alpha \leq 1.3 \%$, the optimal portfolio weights of the MinVar and MinMAD models are $\left\{w_{86}=0.053, w_{129}=\right.$ $\left.0.243, w_{133}=0.704\right\}$ and $\left\{w_{129}=0.219, w_{133}=0.781\right\}$ respectively, as listed in Table 3 . This result is depicted in Figure 2 where funds 86, 129, and 133 are marked by dotted circles, and funds 129 and 133 are marked by dash circles. Since the weight of fund 133 by the MinMAD model is higher than the one by the MinVar model, the dash circle of fund 133 is bigger than the dotted circle.
The optimal portfolios suggested by both models are quite diversified because the locations of the selected funds (funds 86, 129, and 133) are far apart from each other.

Given $\alpha=1.4 \%$ and $1.6 \%$, the Decision Balls for optimal portfolios are shown in Figures 2 and 3. In Figure 2, the optimal portfolio weights by the MinVar and MinMAD models are exactly the same, $\left\{w_{129}=0.413\right.$, $\left.w_{133}=0.587\right\}$. In Figure 3, the optimal portfolio weights by MinVar and MinMAD are $\left\{w_{12}=0.216, w_{129}=0.657\right.$, $\left.w_{133}=0.127\right\}$ and $\left\{w_{12}=0.298, w_{129}=0.598, w_{133}=\right.$ $0.104\}$ respectively.

When comparing Figures 1, 2, and 3, the circle of fund 129 becomes bigger and fund 133's circle becomes smaller when the given expected return $\alpha$ is increased. That is, the optimal portfolio weights shift from the lower to the upper part of the Ball because the funds located in the upper part imply a higher return. Also, there is an obvious cluster, including most of the top 30 funds, except for funds $129,12,133,76$, and 8 . The correlation between the funds can be examined, both visually and directly, through the Decision Balls. Take fund 86 for instance, if the selected fund 86 is not available, funds $121,109,118$, or 90 may be good substitutes because they have a high correlation with fund 86 plus a higher return.

\section{Conclusions}

This study uses open-ended equity funds in Taiwan to in- 


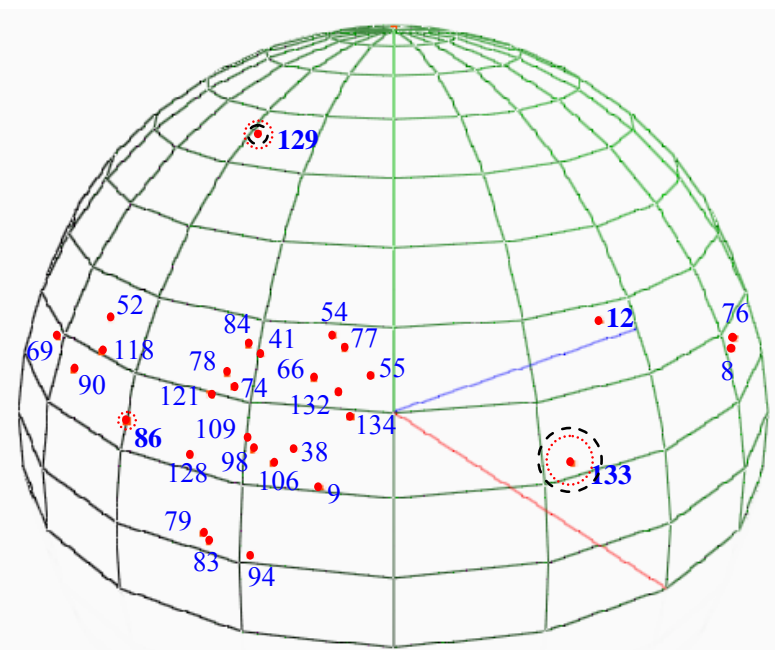

Figure 1. Given $a \leq 1.3 \%$ yields MinVar (dotted circle) and MinMAD (dash circle).

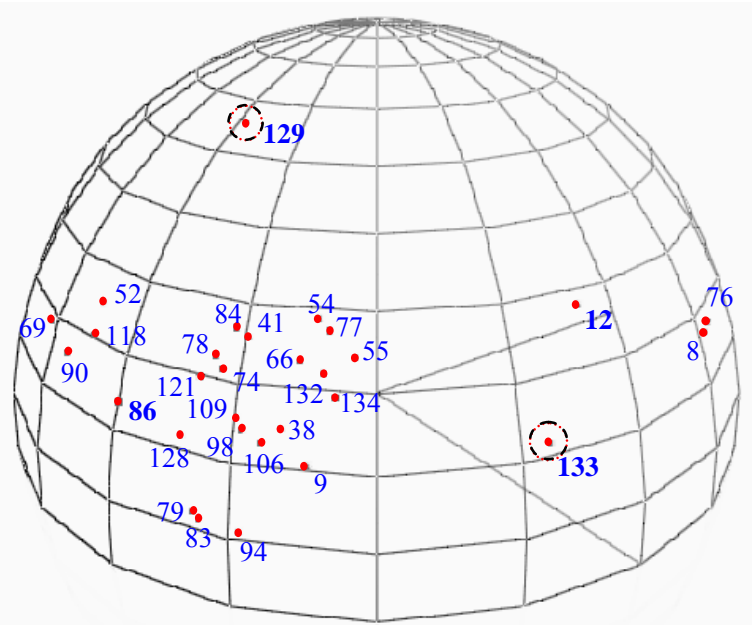

Figure 2. Given $a \leq 1.4 \%$ yields MinVar (dotted circle) and MinMAD (dash circle).

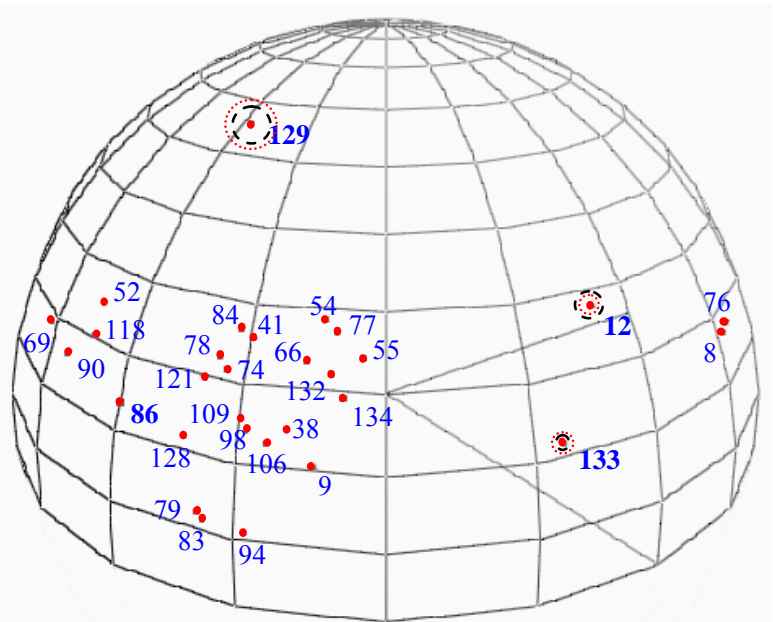

Figure 3. Given $a \leq 1.6 \%$ yields MinVar (dotted circle) and MinMAD (dash circle). 
vestigate three well-known optimal portfolio models, including the mean-variance, maximin, and minimization of mean absolute deviation. The maximin model yields infeasible solutions because all top 30 funds exhibit negative monthly returns in some months during the examined time period. The outcome of the minimization of mean absolute deviation model is quite close to that of the mean-variance model. This result is the same as the conclusions for the study by Papahristodoulou and Dotzauer [18], in which securities traded on the Stockholm Stock Exchange were examined. An extended Decision Ball model is proposed to visualize optimal portfolios on the surface of a sphere, where the distance between two funds indicates the correlation between them, and the fund with a higher return is located closer to the North Pole. The scale of optimal portfolio weights is represented by the size of the circle of the selected fund. By observing the Decision Balls, investors can see the optimal portfolio, compare the optimal weights provided by the different models, view the cluster of funds, and even find substitute funds if the preferred funds are not available. In future studies, the question of how to linearize this non-linear model in order to general a global optimal solution can be addressed.

\section{References}

[1] I. Simonson and A. Tversky, "Choice in Context: Tradeoff Contrast and Extremeness Aversion," Journal of Marketing Research, Vol. 29, No. 3, 1992, pp. 281-295. doi: $10.2307 / 3172740$

[2] L. M. Seiford and J. Zhu, "Context-Dependent Data Envelopment Analysis-Measuring Attractiveness and Progress," Omega, Vol. 31, No. 5, 2003, pp. 397-408. doi:10.1016/S0305-0483(03)00080-X

[3] A. Meyer, "Visual Data in Organizational Research," Organization Science, Vol. 2, No. 2, 1991, pp. 218-236.

[4] H. Markowitz, "Portfolio Selection," Journal of Finance, Vol. 7, No. 1, 1952, pp. 77-91. doi:10.2307/2975974

[5] C. Genest and S. S. Zhang, "A Graphical Analysis of Ratio-Scaled Paired Comparison Data," Management Science, Vol. 42, No. 3, 1996, pp. 335-349. doi: $10.1287 /$ mnsc. 42.3 .335

[6] L. C. Ma and H. L. Li, "Using Gower Plots and Decision Balls to Rank Alternatives Involving Inconsistent Preferences," Decision Support Systems, Vol. 51, No. 3, 2011, pp. 712-719. doi:10.1016/j.dss.2011.04.004

[7] B. P. Gladish, D. F. Jones, M. Tamiz and A. B. Terol, "An Interactive Three-Stage Model for Mutual Funds
Portfolio Selection," Omega, Vol. 35, No. 1, 2007, pp. 75-88. doi:10.1016/j.omega.2005.04.003

[8] H. L. Li and L. C. Ma, "Visualizing Decision Processes on Spheres Based on the Even Swap Concept," Decision Support Systems, Vol. 45, No. 2, 2008, pp. 354-367.

[9] L. C. Ma, "Visualizing Preferences on Spheres for Group Decisions Based on Multiplicative Preference Relations," European Journal of Operational Research, Vol. 203, No. 1, 2010, pp. 176-184. doi:10.1016/j.ejor.2009.07.008

[10] I. Borg and P. Groenen, "Modern Multidimensional Scaling," Springer, New York, 1997.

[11] T. F. Cox and M. A. A. Cox, "Multidimensional Scaling on a Sphere," Communications on Statistics: Theory and Methods, Vol. 20, No. 9, 1991, pp. 2943-2953. doi:10.1080/03610929108830679

[12] W. S. Desarbo and K. Jedidi, "The Spatial Representation Of Heterogeneous Consideration Sets," Marketing Science, Vol. 14, No. 3, pp. 326-342. doi: $10.1287 / \mathrm{mksc} .14 .3 .326$

[13] R. L. Andrews and A. K. Manrai, "MDS Maps For Product Attributes and Market Response: An Application to Scanner Panel Data," Marketing Science, Vol. 18, No. 4, 1999, pp. 584-604. doi:10.1287/mksc.18.4.584

[14] P. G. Shu, Y. H. Yeh and T. Yamada, "The Behavior of Taiwan Mutual Fund Investors-Performance and Fund Flows," Pacific-Basin Finance Journal, Vol. 10, No. 5, 2002, pp. 583-600. doi:10.1016/S0927-538X(02)00070-7

[15] C. S. Hsu and J. R. Lin, "Mutual Fund Performance and Persistence in Taiwan: A Non-Parametric Approach," The Service Industries Journal, Vol. 27, No. 5, 2007, pp. 509-523. doi:10.1080/02642060701411658

[16] M. R. Young, "A Minimax-Portfolio Selection Rule with Linear Programming Solution," Management Science, Vol. 44, No. 5, 1998, pp. 673-683. doi: $10.1287 / \mathrm{mnsc} .44 .5 .673$

[17] H. Konno and H. Yamazaki, "Mean-Absolute Deviation Portfolio Optimization Model and Its Application to Tokyo Stock Market," Management Science, Vol. 37, No. 5, 1991, pp. 519-531. doi:10.1287/mnsc.37.5.519

[18] C. Papahristodoulou, and E. Dotzauer, "Optimal Portfolios Using Linear Programming Models," Journal of the Operational Research Society, Vol. 55, No. 11, 2004, pp. 1169-1177.

[19] T. F. Cox and M. A. A. Cox, "Multidimensional Scaling," CRC Press, Boca Raton, 2000. doi:10.1201/9781420036121

[20] J. B. Kruskal, "Non-Metric Multidimensional Scaling: A Numerical Method," Psychometrica, Vol. 29, No. 2, 1964, pp. 115-129. doi:10.1007/BF02289694 